Article

\title{
Impact of Cyanidin-3-Glucoside on Gut Microbiota and Relationship with Metabolism and Inflammation in High Fat-High Sucrose Diet-Induced Insulin Resistant Mice
}

\author{
Fei Huang ${ }^{1}$, Ruozhi Zhao ${ }^{2}$, Min Xia ${ }^{3}\left(\mathbb{D}\right.$ and Garry X. Shen ${ }^{1,2, *(\mathbb{D}}$ \\ 1 Departments of Food and Human Nutritional Sciences, Internal Medicine, University of Manitoba, \\ Winnipeg, MB R3T2N2, Canada; huangf1@myumanitoba.ca \\ 2 Diabetes Research Group, Department of Internal Medicine, University of Manitoba, 835-715 McDermot Ave, \\ Winnipeg, MB R3E 3P4, Canada; Ruozhi.Zhao@umanitoba.ca \\ 3 School of Public Health, Sun Yat-Sen University, Guangzhou 510275, China; xiamin@mail.sysu.edu.cn \\ * Correspondence: garry.shen@umanitoba.ca; Tel.: +1-204-789-3816; Fax: +1-204-789-3987
}

Received: 22 July 2020; Accepted: 12 August 2020; Published: 14 August 2020

check for updates

\begin{abstract}
The present study assessed the effects of freeze-dried cyanidin-3-glucoside (C3G), an anthocyanin enriched in dark-red berries, compared to Saskatoon berry powder (SBp) on metabolism, inflammatory markers and gut microbiota in high fat-high sucrose (HFHS) diet-induced insulin-resistant mice. Male C57 BL/6J mice received control, HFHS, HFHS + SBp (8.0 g/kg/day) or HFHS + C3G (7.2 mg/kg/day, equivalent C3G in SBp) diet for 11 weeks. The HFHS diet significantly increased plasma levels of glucose, cholesterol, triglycerides, insulin resistance and inflammatory markers. The HFHS + SBp diet increased the Bacteroidetes/Firmicutes (B/F) ratio and relative abundance of Muriculaceae family bacteria in mouse feces detected using $16 \mathrm{~S}$ rRNA gene sequencing. The HFHS + SBp or HFHS + C3G diet attenuated glucose, lipids, insulin resistance and inflammatory markers, and increased the $\mathrm{B} / \mathrm{F}$ ratio and Muriculaceae relative abundance compared to the HFHS diet alone. The relative abundances of Muriculaceae negatively correlated with body weight, glucose, lipids, insulin resistance and inflammatory mediators. Functional predication analysis suggested that the HFHS diet upregulated gut bacteria genes involved in inflammation, and downregulated bacteria involved in metabolism. C3G and SBp partially neutralized HFHS diet-induced alterations of gut bacteria. The results suggest that C3G is a potential prebiotic, mitigating HFHS diet-induced disorders in metabolism, inflammation and gut dysbiosis, and that $\mathrm{C} 3 \mathrm{G}$ contributes to the metabolic beneficial effects of SBp.
\end{abstract}

Keywords: cyanidin-3-glucoside; Saskatoon berry; high fat-high sucrose diet-induced insulin-resistant mice; gut microbiota; inflammation

\section{Introduction}

Diabetes has become one of the most common metabolic disorders worldwide, and the trend of this surge is continuing. Nine out of ten diabetic patients in adults have type 2 diabetes (T2D), which is characterized as insulin resistance and is often associated with obesity [1]. Genetic factors and multiple environmental factors are implicated in the etiology of T2D. However, the precise mechanism or treatment for T2D remains unclear [2]. A Western pattern diet enriched with fat or sugar plays a crucial role in the epidemic of T2D in the world [3]. T2D is associated with low-grade chronic inflammation [4]. Accumulating lines of evidence suggest that gut microbiota plays an important role 
in the development of diabetes, obesity and inflammation [5]. Foods are the most common modulator of gut microbiota. Western pattern diet-induced gut dysbiosis is associated with obesity and T2D [6].

Saskatoon berry (Amelanchier alnifolia Nutt.), a type of fruit-bearing shrub, belongs to the Rosaceae family in Amelanchier genus and is also known as Juneberry and service berry [7]. It natively grows in the southern Yukon, the Prairies in Canada and the west northern regions of the United States of America, and has more recently been planted in Europe [8,9]. Saskatoon berry fruits contain more polyphenols, including anthocyanins, compared to other common berries, such as strawberry or blueberry [10]. At least four types of anthocyanins have been detected in Saskatoon berries, among which cyanidin-3-galactoside and cyanidin-3-glucoside (C3G) are most abundant, accounting for about $61-74 \%$ and $18-21 \%$, respectively $[8,11]$.

Saskatoon berry fruits possess abundant antioxidant capacity [12,13]. Previous studies by our group demonstrated that Saskatoon berry powder (SBp) was capable of reducing vascular inflammation in leptin receptor-knockout $(\mathrm{db} / \mathrm{db})$ diabetic mice. Supplementation of C3G resulted in 3-times higher anti-inflammation activity than C3Ga in cultured endothelial cells [11,14]. Our recent study demonstrated that supplementation of $5 \%$ SBp $(\sim 8.0 \mathrm{~g} / \mathrm{kg} /$ day) attenuated high fat-high sucrose (HFHS) diet-induced hyperglycemia, hyperlipidemia, insulin resistance, inflammation and gut dysbiosis in mice [15]. However, the effect of the administration of C3G on the composition of gut microbiota of mice fed with a HFHS diet, and the relationship between the changes in gut microbiota, metabolism and inflammatory mediators in mice receiving a HFHS diet supplemented with C3G have not been documented.

The present study compared the effects of supplementation of C3G and SBp on circulating glucose, lipids, insulin, inflammatory markers and gut microbiota in a HFHS diet-sensitive rodent model, C57 BL/6J mice. Differences in the impact of C3G and SBp supplementation on gut microbial profile and the potential function of the microbial genes in mice fed with a HFHS diet were further investigated using $16 \mathrm{~S}$ ribosome ribonucleic acid (rRNA) gene sequencing and bioinformatics approaches.

\section{Materials and Methods}

\subsection{Animals}

Male C57 BL/6J mice ( $n=32,6$ weeks of age) were obtained from the Jackson Laboratory (Bar Harbor, ME, USA). Mice were housed in standard plastic cages in an air-conditioned room with an alternative $12 \mathrm{~h}$ day/night light cycle and received regular mouse chow and tap water for 1 week to stabilize. The protocols of animal experiments have been approved by the Animal Protocol and Ethics Committee in the University of Manitoba.

\subsection{Dietary Intervention}

Mice were randomized into 4 groups ( $n=8 /$ group, four in a cage) and received one of following diets for 11 weeks: (1) control group receiving a D12450K control diet from Research Diets (New Brunswick, NJ, USA), containing $4.3 \%$ of diet, $19.2 \%$ of protein and $67.3 \%$ of carbohydrates in dry mass without an addition of sucrose, (2) the HFHS group fed with a HFHS diet (D12492, Research Diets) containing 35\% of fat, $26 \%$ of protein and $26 \%$ of carbohydrates, including $9 \%$ sucrose, (3) the SBp group receiving a HFHS diet supplemented with $8.0 \mathrm{~g} / \mathrm{kg}$ body weight of SBp daily and (4) the C3G group fed with a HFHS diet supplemented with $7.2 \mathrm{mg} / \mathrm{kg} /$ day of C3G (equal to the amount of C3G containing $8.0 \mathrm{~g} / \mathrm{kg}$ SBp). SBp was prepared from lyophilized Smoky Saskatoon berries obtained from the Prairie Lane Saskatoon (Portage, MB, Canada) and stored at $-80^{\circ} \mathrm{C}$ [11]. Purified C3G was obtained from Polyphenols (Sandnes, Norway).

\subsection{Animal Monitoring and Sample Collection}

Body weights and food intake of animals was assessed every 2 weeks until 10 weeks after the start of the dietary experiment. Blood $(200 \mu \mathrm{L})$ was collected from mouse saphenous veins every 2 weeks 
after an overnight fasting for measuring levels of plasma glucose to monitor the development of diabetes. Mice were euthanized at 11 weeks after the onset of the dietary experiment via the inhalation of $5 \%$ isoflurane to reduce the pain of the animals. Blood was withdrawn subsequently through heart puncture.

\subsection{Analyses of Metabolic Variables}

The levels of plasma glucose and cholesterol of mice were analyzed using Sekisui Diagnostics SL reagent kits (Charlottetown, PE, Canada). Plasma levels of triglycerides were measured using BioAssay Systems reagents (Hayward, CA, USA). Insulin levels in plasma were assessed using enzyme-linked immunosorbent assay (ELISA) kits from EMD Millipore (Billerica, MA, USA) for insulin. The homeostatic model assessment of insulin resistance (HOMA-IR) of the mice was calculated from plasma insulin and glucose from simultaneously withdrawn blood samples as previously described [16].

\subsection{Measurement of Circulating Inflammatory Markers}

Monocyte chemotactic protein-1 (MCP-1) and plasminogen activator inhibitor-1 (PAI-1) were measured using ELISA kits from Thermo Fisher Scientific (Ottawa, ON, Canada) for MCP-1 and Oxford Biomedical Research (Oxford, MI, USA) for PAI-1, respectively.

\subsection{Fecal Sample Collection}

Mice were housed in a singly housed cage with fresh bedding overnight at the 10th week after the start of the dietary experiment. Fecal pellets were collected from individual cages and stored in separated tubes at $-80^{\circ} \mathrm{C}$ before further analysis.

\subsection{Extraction and Sequencing of Bacteria DNA}

DNA extraction was achieved using a PowerFecal DNA Isolation Kit (QIAGEN, Germantown, MD). DNA was quantified using a NanoDrop 2000 spectrophotometer (Thermo Scientific, Boston, MA, USA). Bacteria DNA in mouse feces was amplified using polymerase chain reaction (PCR) with modified primers containing 515F (5'-GTGYCAGCMGCCGCGGTAA) and 926R (5'-CCGYCAATTYMTTTRAGTTT) targeting the V4-V5 region of bacterial DNA. A high-throughput Hamilton Nimbus Select robot and Coastal Genomics analytical gels were run to verify the quality of PCR products. Failed amplicons with spurious bands were repeatedly amplified after modifying conditions for PCR until qualified bands were produced. The PCR amplicons were normalized by using a high-throughput Charm Biotech Just-a-Plate 96-well normalization kit, then pooled to construct a library and quantified before sequencing on an Illumina MiSeq platform in the Integrated Microbiome Resource in the Dalhousie University [17].

\subsection{Bioinformatic Analysis and Statistics}

Freshly generated raw data of gut microbiota in the form of a fastq file was demultiplexed according to the barcode sequences, followed by trimming using Cutadapt (version 1.17) to remove primers. Trimmed reads were imported as artifacts into an open-source bioinformatics pipeline of decentralized microbiome analysis package Quantitative Insights Into Microbial Ecology 2 (QIIME2, version: 2018. 8) [18]. After joining of paired-end reads and filtering out of low-quality reads, amplicon sequence variants (ASVs) were obtained through DADA2 workflow. Taxonomies were assigned to ASVs using a Naive-Bayes approach and SILVA database. Diversity metrics (Core-metrics-phylogenetic) within QIIME2 were used for evaluating $\alpha$ - and $\beta$-diversity. Differences between data from multiple groups were examined using the analysis of variance (ANOVA) and post-hoc Tukey test. Significant difference was set at $p<0.05$. Relative abundances of ASVs assigned with taxonomy in the feature table were correlated with physical and clinical parameters. The Phylogenetic Investigation of Communities by Reconstruction of Unobserved States (PICRUSt) was used to identify differences in predictive 
metagenome function. Functions of microbial genes were predicted with the use of Galaxy web application, and Kyoto Encyclopedia of Genes and Genomes [19].

\section{Results}

\subsection{Effects of HFHS Diet and Supplementation of C3G on Body Weights and Food Intake of Mice}

Significant increases in body weights between mice fed with the control diet and the HFHS diet with and without a supplementation of SBp or C3G were detected at $\geq 2$ weeks after the start of the intervention $(p<0.05$ or 0.01$)$. The trend of body weight increase in mice receiving the HFHS diet continued throughout the experiment. No significant difference was detected among mice fed with the HFHS diet with or without a supplementation with SBp or C3G (Figure 1A). Daily food intake of all mice was measured at onset and every 2 weeks during the dietary experiment until the 10th week after the start of the dietary intervention. No significant difference in food intake was detected among various groups receiving different experimental diets in the present study (Figure 1B).

A

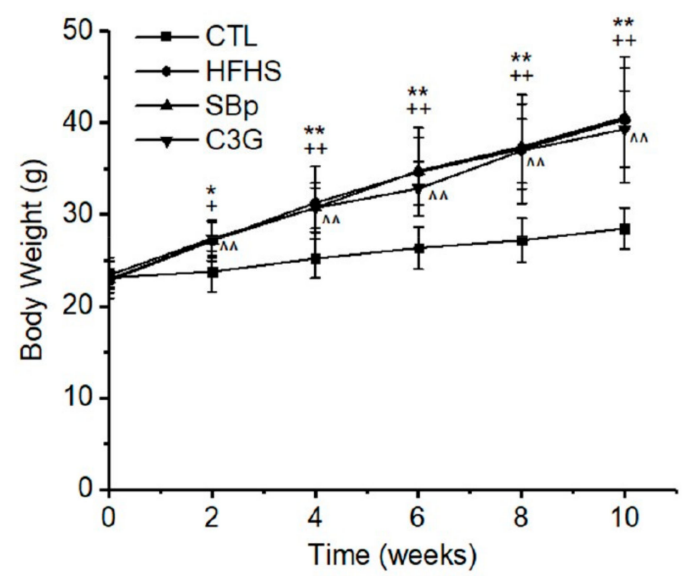

B

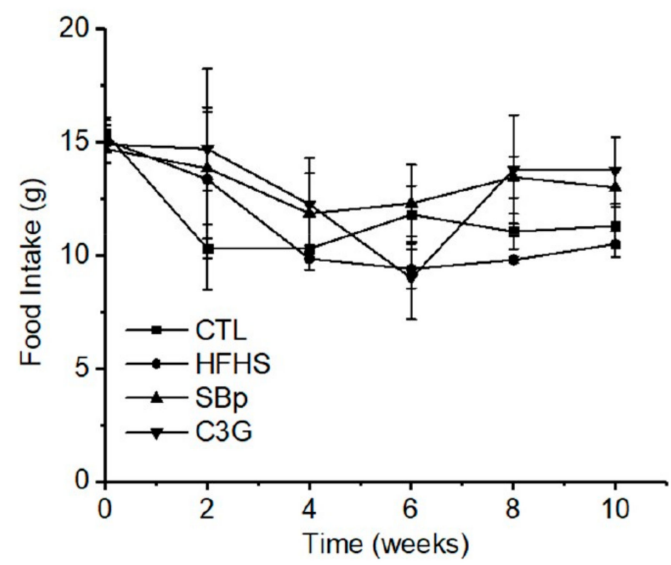

Figure 1. Effects of Cyanidin-3-glucoside (C3G) supplemented in a high fat-high sucrose (HFHS) diet on the body weight and food intake of mice. Male C57 BL/J6 mice (6 weeks of age) were randomized into 4 groups and received the following diets for 11 weeks: control (CTL) group: control diet, HFHS group: HFHS diet, Saskatoon berry powder (SBp) group: SBp $(8.0 \mathrm{~g} / \mathrm{kg} /$ day $)$ supplemented in the HFHS diet, C3G group: C3G $(7.2 \mathrm{mg} / \mathrm{kg} /$ day) supplemented in the HFHS diet. Body weights and food intake were measured every two weeks up to 10 weeks. (A) Body weights, (B) daily food intake. The values are expressed as mean \pm standard deviation (SD) g $\left(n=8\right.$ /group). ${ }^{*}, * *: p<0.05$ or 0.01 HFHS versus CTL group,,$+++: p<0.05$ or $0.01 \mathrm{SBp}$ versus CTL group, ${ }^{\wedge}{ }^{\wedge}: p<0.01 \mathrm{C} 3 \mathrm{G}$ versus CTL group.

\subsection{Effects of C3G on Glucose and Lipid Metabolism in HFHS Diet-Induced Obese Mice}

The HFHS diet significantly increased the levels of fasting plasma glucose (FPG) in mice compared to the control diet $(p<0.01)$. The supplementation with SBp or C3G in the HFHS diet significantly lowered FPG, compared to that in HFHS alone $(p<0.05$ or 0.01$)$. However, the levels of FPG in the SBp or $\mathrm{C} 3 \mathrm{G}$ group were still significantly higher than that in the control group. No significant difference in FPG was found between the SBp and C3G groups. The HFHS diet also significantly elevated the levels of cholesterol and triglycerides in plasma compared to the control diet $(p<0.01)$. Plasma cholesterol or triglyceride levels in both the SBp and C3G groups were significantly lower than that in the HFHS group $(p<0.01)$. Cholesterol and triglyceride levels in the plasma of mice receiving the HFHS diet supplemented with SBp or C3G were significantly higher than those in the control group $(p<0.01)$. No significant difference in plasma cholesterol or triglycerides was detected between the SBp and C3G groups (Figure 2). 


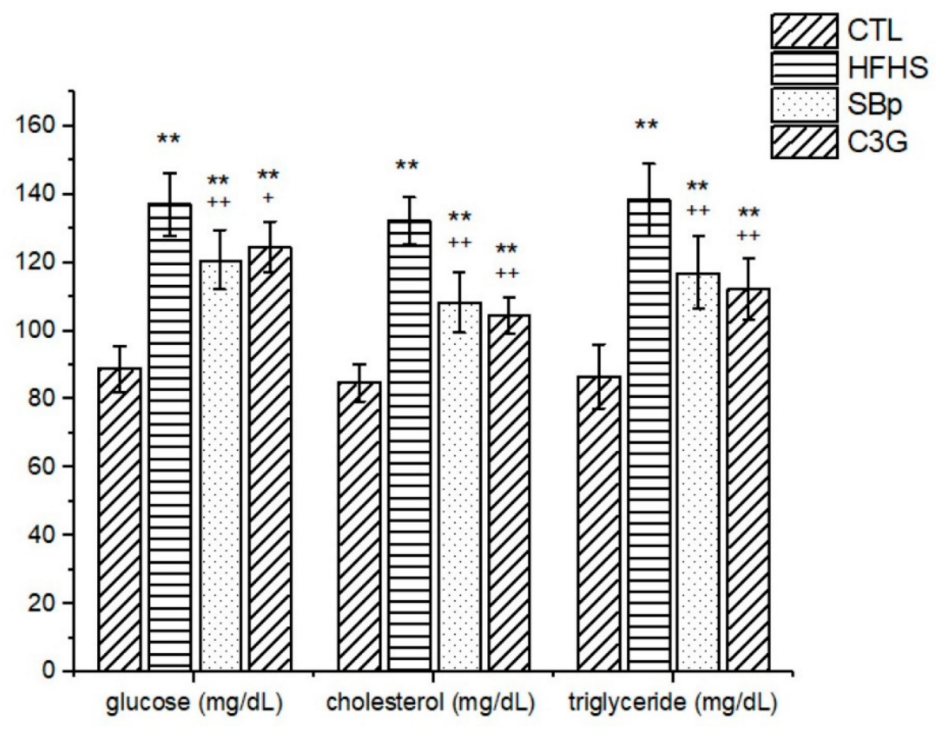

Figure 2. Levels of glucose, cholesterol and triglycerides in plasma of mice fed with HFHS diets supplemented with or without C3G. The dietary regimen was the same as described in the legend of Figure 1. Fasting plasma glucose, cholesterol and triglycerides were measured biochemically using assay kits. Values are expressed as mean $\pm \mathrm{SD} \mathrm{mg} / \mathrm{dL}\left(n=8 /\right.$ group). ${ }^{* *}: p<0.01$ versus the control (CTL) group;,$+++: p<0.05$ or 0.01 versus the HFHS group.

\subsection{Effects of C3G on Fasting Plasma Insulin and Insulin Resistance in Mice Receiving the HFHS Diet}

Insulin resistance was assessed using HOMA-IR generated from the levels of FPG and insulin in the plasma samples simultaneously withdrawn from mice. The levels of plasma insulin and HOMA-IR in the HFHS group were significantly higher than that in the control group $(p<0.01)$. Supplementation with SBp and C3G significantly reduced the levels of insulin and HOMA-IR compared to that in mice fed with the HFHS diet alone $(p<0.01)$. The levels of insulin and HOMA-IR in the SBp or C3G group were still higher than that in the control group $(p<0.01)$. No significant difference in insulin or HOMA-IR was detected between the SBp and C3G groups (Figure 3A,B).

A

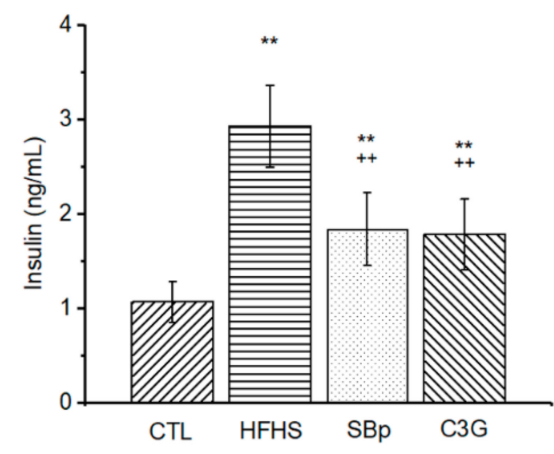

B

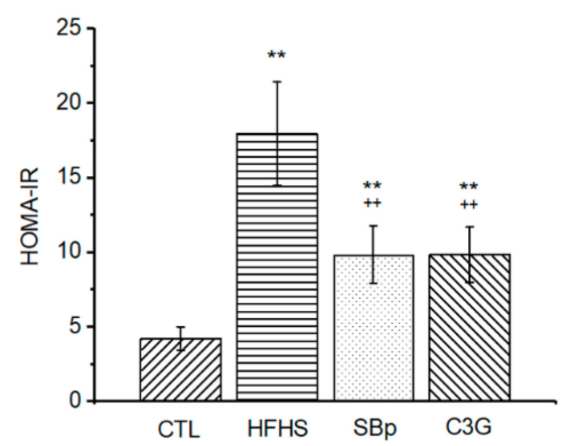

Figure 3. Effects of HFHS diets supplemented with C3G on insulin and insulin resistance in mice. The experimental regimen was described in the legend of Figure 1. The levels of fasting plasma insulin $(\mathrm{ng} / \mathrm{mL})$ were measured at indicated time points $(\mathbf{A})$. Homeostatic model assessment of insulin resistance (HOMA-IR) was calculated according to the levels of glucose and insulin in the same plasma samples (B). Values are expressed as mean $\pm \mathrm{SD}\left(n=8\right.$ /group). ${ }^{* *}: p<0.01$ versus the control (CTL) group; ++: $p<0.05$ or 0.01 versus the HFHS group. 


\subsection{Effects of HFHS Diet and the Supplementation of C3G on Circulating Inflammatory Markers in Mice}

The HFHS diet elevated the levels of MCP-1 and PAI-1, two inflammatory markers, in the plasma of mice $(p<0.01)$. The supplementation of SBp or C3G in the HFHS diet significantly reduced the circulating levels of MCP-1 and PAI-1 compared to the HFHS diet in mice $(p<0.01)$. The levels of the inflammatory markers in mice receiving the SBp or C3G diets were still significantly higher than that in the control group $(p<0.01)$. No significant difference in plasma MCP-1 or PAI-1 was detected between mice receiving the HFHS diet supplemented with SBp or C3G (Figure 4A,B).

A

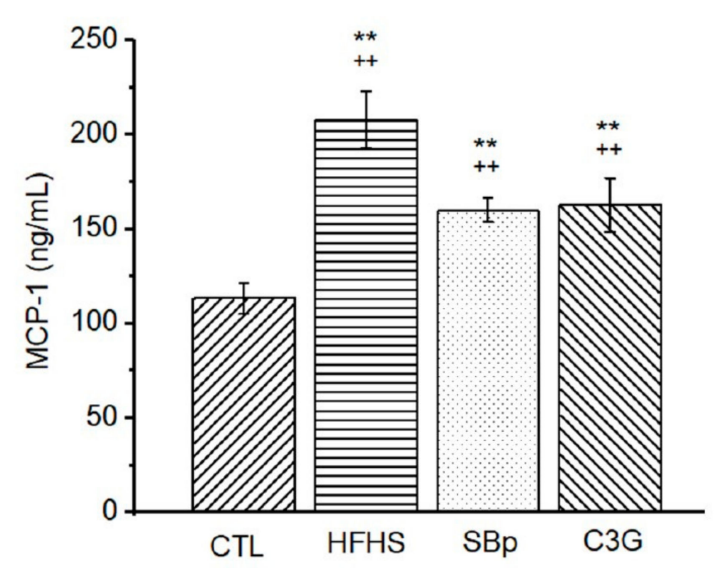

B

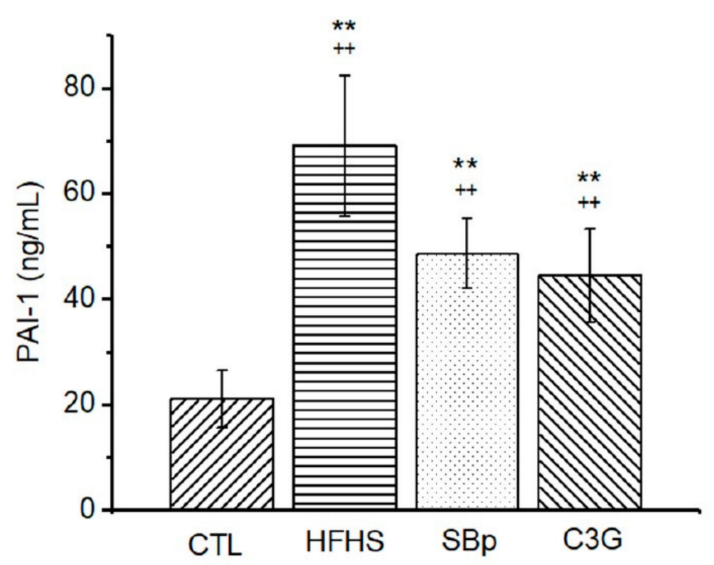

Figure 4. Levels of inflammatory regulators in plasma of mice receiving the HFHS diet supplemented with C3G. The experimental regimen was described in the legend of Figure 1. The levels of monocyte chemotactic protein-1 (MCP-1) and plasminogen activator inhibitor-1 (PAI-1) were analyzed in plasma collected before tissue harvesting using enzyme-linked immunosorbent assay (ELISA) kits for mouse MCP-1 (A) or PAI-1 (B). Values are expressed as mean \pm SD ng/mL ( $n=8 /$ group). ${ }^{* *}: p<0.01$ versus the control (CTL) group; ++: $p<0.05$ or 0.01 versus the HFHS group.

\subsection{Impact of C3G Supplementation on Gut Microbiota in HFHS Diet-Fed Mice}

The results of $\beta$-diversity analysis demonstrated that the gut microbial compositions in the stool of mice from the four dietary groups were well separated in principal component analysis (Figure 5). No significant difference in $\alpha$-diversity variables was detected in mice receiving different diets (Shannon index, chao1). Table 1 demonstrates that Bacteroidetes and Firmicutes represented the vast majority phylum bacteria in mouse feces. Mice fed with the HFHS diet had lower abundances of Bacteroidetes, Actinobacteria, Proteobacteria and Verrucomicrobia phylum bacteria, but a higher relative abundance of Firmicutes phylum bacteria compared to mice receiving the control diet $(p<0.05$ or 0.01 ). Supplementation with SBp or C3G to the HFHS diet augmented the relative abundance of Bacteroidetes and reduced that of Firmicutes in mice feces $(p<0.01)$. The relative abundance of Actinobacteria in the stool of the C3G group was the only type of phylum bacteria significantly different from that in SBp group $(p<0.05)$. 


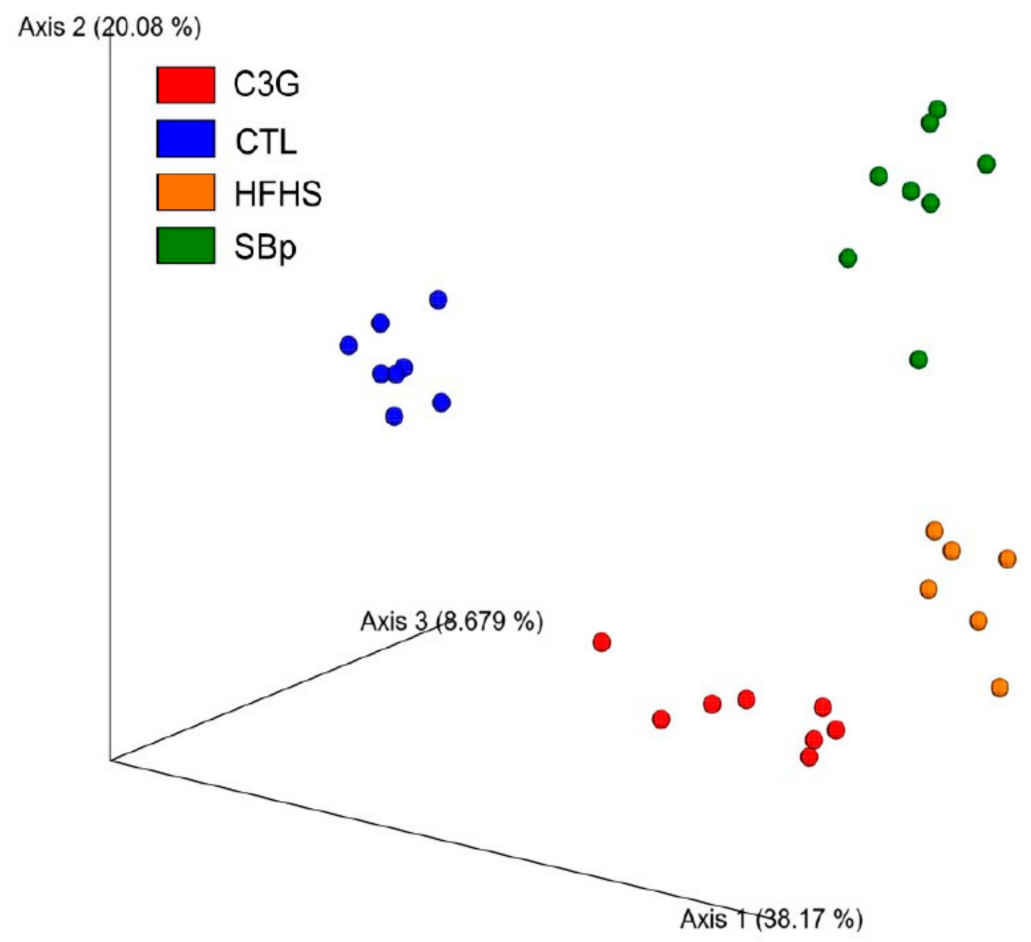

Figure 5. Effect of the HFHS diet supplemented with C3G on $\beta$-diversity of gut microbiota in mice. The experimental regimen was described in the legend of Figure 1. Principle component analysis (PCA) was based on Bray-Curtis dissimilarities between all sample sets (weighted by taxon abundance).

Table 1. Effect of different diets on the abundances of gut microbiota on phylum level.

\begin{tabular}{ccccc}
\hline Phylum Bacteria & CTL (\%) & HFHS (\%) & SBp (\%) & C3G (\%) \\
\hline Actinobacteria & $5.66 \pm 2.75$ & $0.26 \pm 0.11^{* *}$ & $0.24 \pm 0.15^{* *}$ & $2.85 \pm 1.83^{*},+$, \\
Bacteroidetes & $54.28 \pm 6.91$ & $34.25 \pm 7.36^{* *}$ & $55.80 \pm 6.95++$ & $48.36 \pm 4.31^{*+}$ \\
Firmicutes & $23.59 \pm 6.41$ & $59.53 \pm 8.00^{* *}$ & $36.01 \pm 5.73^{* *},++$ & $42.34 \pm 4.63^{* *},++$ \\
Proteobacteria & $2.36 \pm 0.71$ & $0.68 \pm 0.20^{* *}$ & $0.81 \pm 0.21^{* *}$ & $0.63 \pm 0.26^{* *}$ \\
Tenericutes & $0.21 \pm 0.23$ & $0.20 \pm 0.14$ & $0.24 \pm 0.16$ & $0.46 \pm 0.34$ \\
Verrucomicrobia & $13.88 \pm 3.81$ & $4.96 \pm 3.23^{* *}$ & $6.78 \pm 2.27^{* *}$ & $5.29 \pm 1.81^{* *}$ \\
Others & $0.02 \pm 0.02$ & $0.12 \pm 0.09^{*}$ & $0.11 \pm 0.09$ & $0.08 \pm 0.03$ \\
\hline
\end{tabular}

Male C57 BL/J6 mice (6 weeks of age) were randomized into 4 groups and received following diets for 11 weeks: control (CTL) group: low-fat diet; HFHS group: HFHS diet; SBp group: SBp (8.0 g/kg/day) supplemented in the HFHS diet; C3G group: C3G (7.2 mg/kg/day) supplemented in the HFHS diet. Values in the tables were expressed in mean $\pm \mathrm{SD}$ (\% of total gut microbiota, $n=8 /$ group). ${ }^{*},{ }^{* *}: p<0.05$ or 0.01 versus control group;,$+++: p<0.05$ or 0.01 versus HFHS group; ${ }^{\wedge}: p<0.05$ versus SBp group.

The relative abundance of Bacteroidetes phylum bacteria in mice with HFHS diets was significantly lower than that in mice fed with the other three types of diets $(p<0.01$, Figure 6A). The relative abundance of Firmicutes phylum bacteria in the HFHS group was significantly higher compared to that in the other three groups ( $p<0.01$, Figure $6 \mathrm{~B}$ ). The HFHS group also had a significantly lower ratio of Bacteroidetes/Firmicutes (B/F) and a higher Firmicutes/Bacteroidetes ratio compared to that in the control, SBp or C3G groups $(p<0.05$ or 0.01$)$. The SBp group had a significantly higher $\mathrm{B} / \mathrm{F}$ ratio and lower $\mathrm{F} / \mathrm{B}$ ratio compared to the HFHS group. C3G supplementation induced a significantly lower $\mathrm{F} / \mathrm{B}$ ratio $(p<0.01)$, but not B/F ratio, compared to the HFHS group (Figure $6 \mathrm{C}, \mathrm{D}$ ). 
A

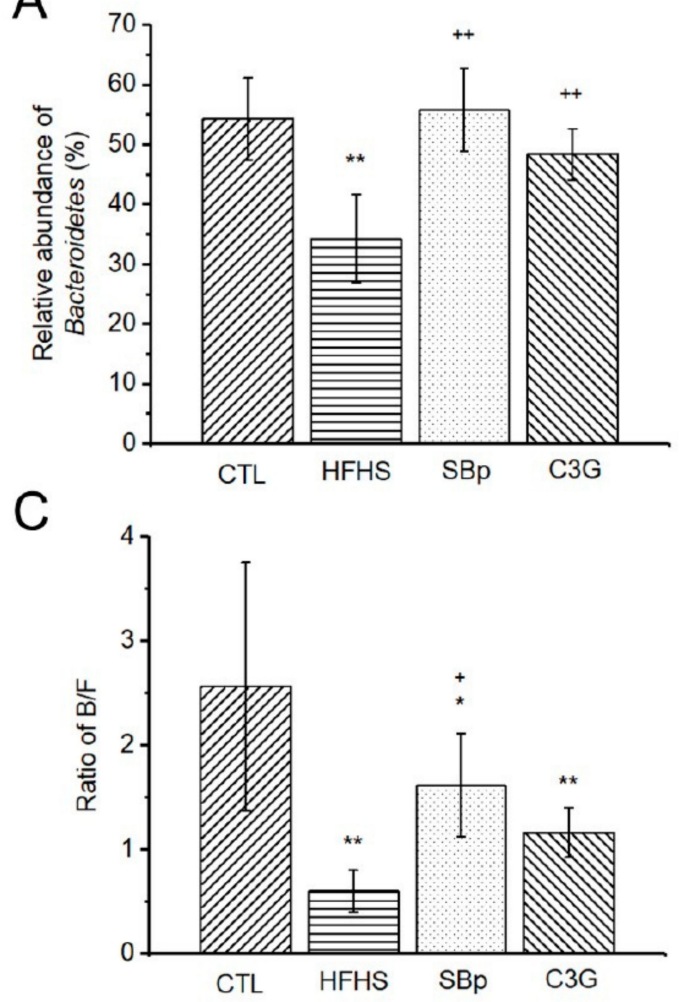

B

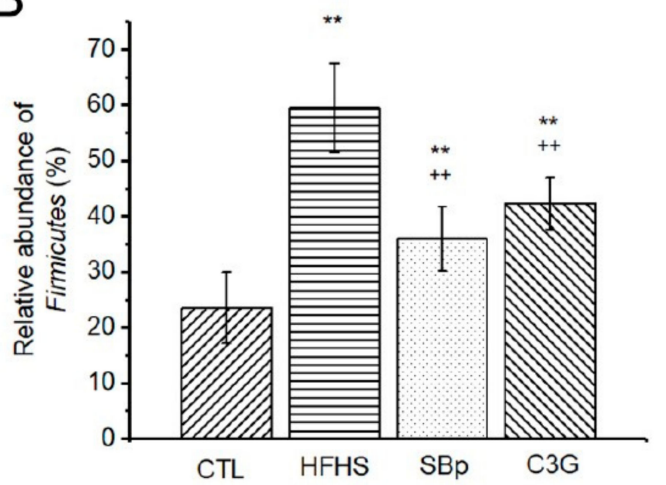

$\mathrm{D}$

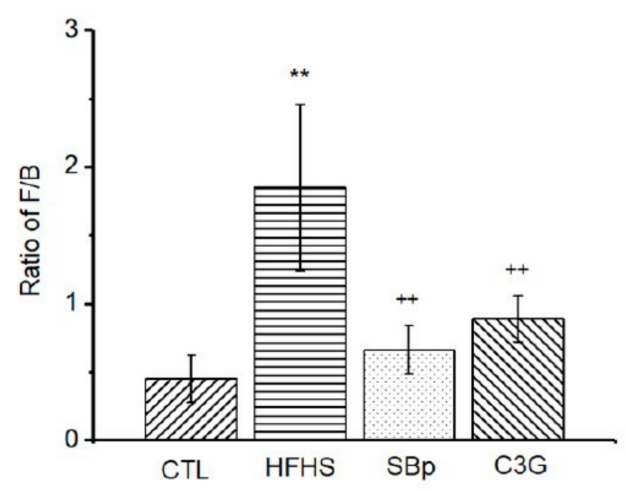

Figure 6. Effects of HFHS diet supplemented with or without the relative abundance of Bacteroidetes and Firmicutes and their ratios. The experimental regimen was described in the legend of Figure 1. (A) Relative abundance (\%) of Bacteroidetes in gut microbial composition. (B) Relative abundance (\%) of Firmicutes in gut microbial composition. (C) Ratio of Bacteroidetes over Firmicutes (B/F) in gut microbiota. (D) Ratio of Firmicutes over Bacteroidetes (F/B) in gut microbiota. Values are expressed as mean \pm SD (\%) ( $n=8$ /group). ${ }^{*}, * *: p<0.05$ or 0.01 versus the control (CTL) group;,$+++: p<0.05$ or 0.01 versus the HFHS group.

Statistical differences in the relative abundances of all types of gut family bacteria, except that of Bifidobacteriaceae, were detected among mice receiving various diets $(p<0.01$, Figure $7 \mathrm{~A})$. Mice fed with the HFHS diet had evidently higher relative abundances in Erysipelotrichaceae or Lachnospiraceae and lower relative abundance in Muribaculaceae family bacteria compared to that in the other groups (heatmap in Figure 7A). Supplementation of SBp or C3G decreased the relative abundance of Lachinospiraceae, and increased that of Muribacculaceae compared to HFHS diet $(p<0.05)$. However, the relative abundances of several family bacteria in the SBp group were different from that in the C3G group (see open triangles in Figure 7A). The heatmap (Figure 7B, right) demonstrated correlations between the relative abundance of family bacteria and some diabetes-related biochemical parameters. The abundances of Defluviitaleaceae, Eggerthellaceae, Erysipelotrichaceae, Family XIII, Lachnospiraceae, Peptpcoccaceae, Peptostreptococcaeae, Rununococcaceae and Streptococcaceae family bacteria were positively correlated with body weights, glucose/lipid metabolism and inflammatory markers in the mice, while Clostridiaceae 1, Clostridiales vadinBB60 group, Muribaculaceae and Lactobacillaceae were negatively correlated with the physical, metabolic and inflammatory variables. The relative abundance of Muribaculaceae in mice in the HFHS group was the lowest among all groups. Both SBp and C3G supplementations significantly increased the relative abundances of Muribaculaceae family bacteria compared to the HFHS group. 


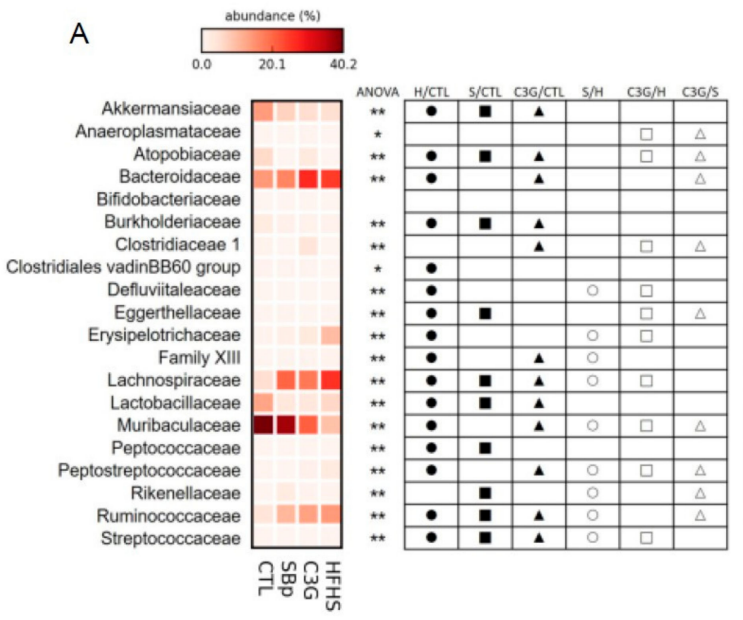

B

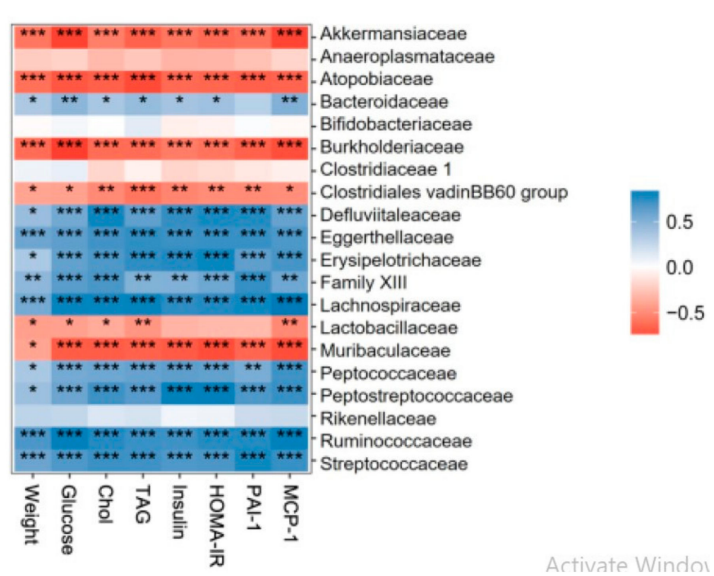

C

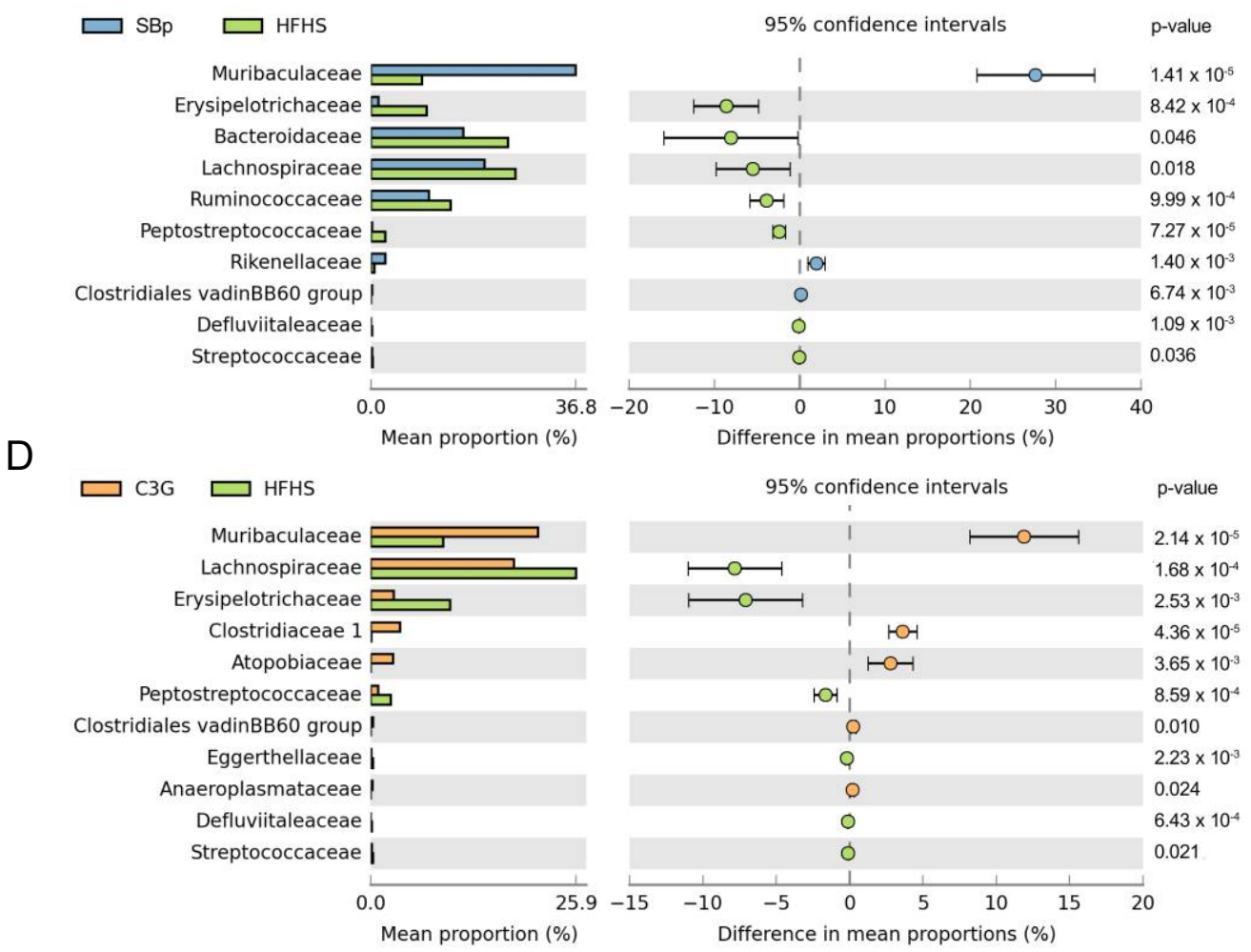

Figure 7. Effect of four HFHS (H) diets supplemented with C3G on the relative abundance of gut family bacteria. The experimental regimen was described in the legend of Figure 1. (A) Statistical differences among mice with different diets (analysis of variance (ANOVA) and post-hoc Tukey test), (B) correlation heatmap of relative abundance of gut microbiota on family level with physiological and biochemical parameters, (C) extended error bar plot (STAMP tool) showing difference in mean relative abundance between the SBp (S) group and HFHS group, (D) mean proportion and difference in mean proportion of family bacteria (STAMP tool) between the $\mathrm{C} 3 \mathrm{G}$ group and the HFHS group (mean $\pm \mathrm{SD}$ ). $*: p<0.05$ in overall ANOVA result, $\bullet: p<0.05$ in the HFHS (H) group versus the control (CTL) group $(\mathrm{H} / \mathrm{CTL}), \mathbf{\square}: p<0.05$ in the SBp (S) group versus the CTL group (S/CTL), $\mathbf{\Delta}: p<0.05$ in the C3G group versus the CTL group (C3G/CTL), $\bigcirc: p<0.05$ in the $\mathrm{S}$ group versus the $\mathrm{H}$ group $(\mathrm{S} / \mathrm{H}), \square: p<0.05$ in the $\mathrm{C} 3 \mathrm{G}$ group versus the $\mathrm{H}$ group $(\mathrm{C} 3 \mathrm{G} / \mathrm{H}), \Delta: p<0.05$ in the $\mathrm{C} 3 \mathrm{G}$ group versus the $\mathrm{S}$ group $(\mathrm{C} 3 \mathrm{G} / \mathrm{S})$. $*, * *, * * *: p<0.05$ or 0.01 or 0.001 in positive (blue) or negative (red) correlations between the abundance of each gut family bacteria and physiological or biochemical variables. 
The greatest difference in the mean proportion of family bacteria between the HFHS group and the SBp or C3G groups was Muribaculaceae family bacteria (Figure 7C,D). The mean proportion of Muribaculaceae in the HFHS group was significantly lower than that in the SBp or C3G groups $(p<0.001)$. The mean proportion of Lachnospiraceae was significantly higher in the HFHS group and lower in the SBp and C3G groups ( $p<0.05$ or 0.001). A similar pattern of changes was detected in the mean proportion of Erysipelotrichaceae, which was higher in the HFHS group but lower in the SBp and C3G group. The mean proportion of Ruminococcaceae was significantly higher in the HFHS group compared to the SBp group $(p<0.001$, Figure $7 C)$, while no significant difference in the mean proportion of Ruminococcaceae was detected between the HFHS and C3G groups (Figure 7D).

\subsection{Functional Predication of the Changes in Gut Microbial Genes Induced by the HFHS Diet with and without C3G Supplementation}

Function prediction using PICRUSt analysis demonstrated the impact of the HFHS diet and that supplemented with SBp or C3G on the relative abundances of genes of gut microbes in 15 classes of predicted functions in mice $(p<0.01$, ANOVA, Figure 8$)$. Microbial genes related to membrane transport were the most abundant in the stool of mice in the present study. At least two types of ASV (operational taxonomic unit or OTUs) distribution among the cellular functions in mice receiving different dietary intervention were found: (1) the HFHS diet resulted in the highest microbial abundances compared to that in the control group, and the supplementation with SBp or C3G attenuated the effect of the HFHS diet, and (2) mice in the control group had more ASVs than in the HFHS group, and the supplementation of SBp or C3G substantially increased ASVs compared to the HFHS diet, but still relatively less than that in the control group. Examples for the first type of cellular functions include membrane transport and cell motility, which may be implicated in inflammation. The second type of cellular functions included the metabolism for carbohydrates, amino acids, lipids, cofactors, vitamins, energy generation, replication and repair, which may potentially contribute to metabolism. It is noticed that transcription was activated, but the translation pathway was suppressed in mice receiving the HFHS diet. SBp or C3G supplementation attenuated the effect of the HFHS diet in transcription or translation in mice. The findings suggest that SBp and C3G may neutralize the effect of the HFHS diet in inflammation and promote metabolism in mice via the modulation of gut microbiota.

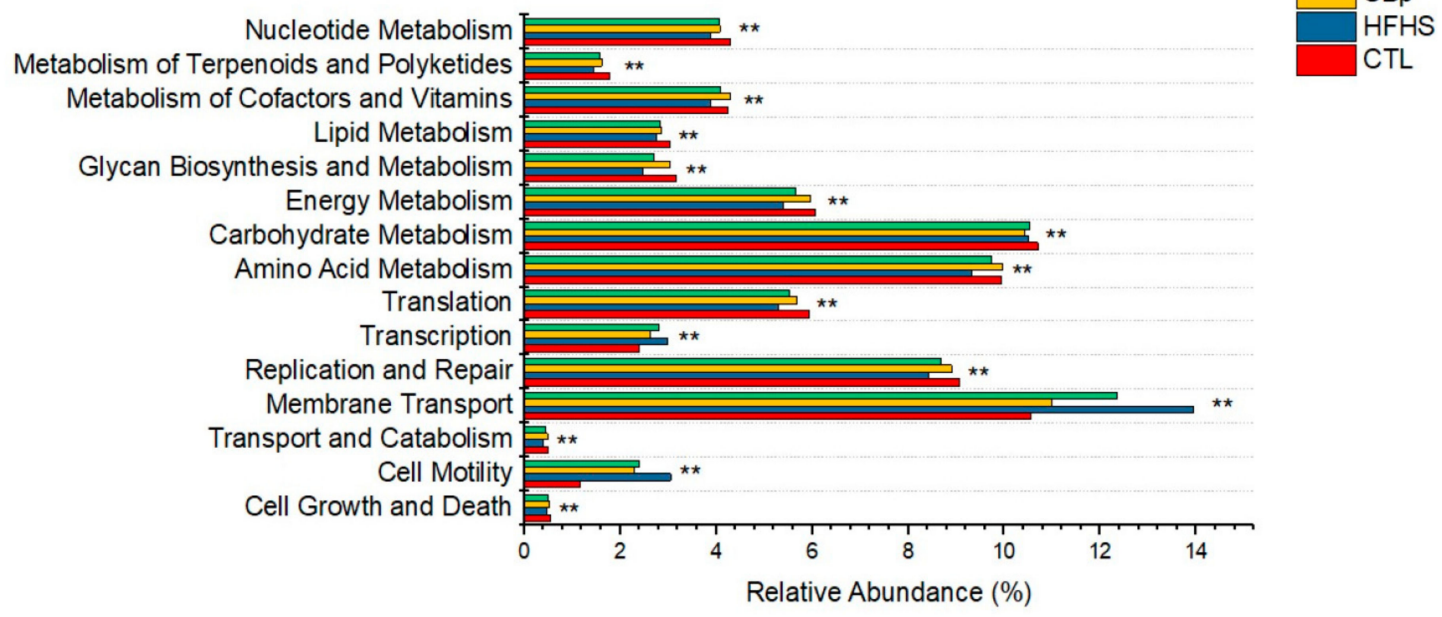

Figure 8. Effects of HFHS diets supplemented with and without C3G on metagenome functional activity in gut microbiota based on the Phylogenetic Investigation of Communities by Reconstruction of Unobserved States (PICRUSt). The experimental regimen was the same as described in legend of Figure 1. Differences in relative abundance (\%) in each selected pathway among various dietary groups are in the form of a bar plot. Values are expressed as mean value ( $n=8 /$ group). ${ }^{* *}: p<0.01$, showing ANOVA results among the four groups. 


\section{Discussion}

The major novel findings generated from the present study include: (1) the supplementation of C3G significantly reduced HFHS diet-induced hyperglycemia, hypercholesterolemia, hypertriglyceridemia, insulin resistance, inflammatory markers and gut dysbiosis in mice in similar extents as those treated with SBp containing a comparable amount of C3G, (2) the HFHS diet supplemented with C3G increased the abundance of Bacteroidetes phylum bacteria and decreased the abundance of Firmicutes phylum bacteria compared to the HFHS diet alone, (3) C3G supplementation increased the abundance of gut Muribaculaceae family bacteria in comparable intensity as SBp supplementation, but the influence of C3G on several other types of family bacteria differed from that in mice receiving SBp. The abundance of Muribaculaceae bacteria in mouse feces was negatively correlated with body weights, FPG, lipids and inflammatory markers in the mice. (4) The results of functional prediction analysis suggest that the supplementation of $\mathrm{C} 3 \mathrm{G}$ reduced the abundance of gut microbial genes involved in inflammation and enhanced gut microbial genes involved in the metabolic processes in mice receiving the HFHS diet.

Previous studies demonstrated that C3G increased the translocation of glucose transporter-4 in skeletal muscle through the activation of insulin and the AMP-activated protein kinase pathway in mice [20]. C3G also augmented glucose-induced insulin secretion in INS-1E pancreatic $\beta$-cells and glucose uptake in HepG2 hepatocytes [21]. C3G inhibited high-glucose-induced cholesterol accumulation in HK-2 kidney epithelial cells through the activation of the LXR $\alpha$ pathway [22]. C3G-rich Bayberry extract reduced mitochondrial reactive oxygen species production and necrosis of INS-1 cells, and lowered blood glucose in streptozotocin-induced diabetic mice [23]. Previous studies in our laboratory demonstrated that C3G inhibited glycated low-density lipoproteins-induced NADPH oxidase activation, mitochondrial dysfunction and cell viability in cultured vascular endothelial cells [24]. The inhibitory effect of C3G on endoplasmic reticulum stress markers in endothelial cells was at least 3-times stronger than the most abundant anthocyanin, cyandin-3-galacotoside, in SBp [14]. SBp supplementation significantly reduced HFHS diet-induced hyperglycemia, hypercholesterolemia, hypertriglyceridemia and insulin resistance in C57 BL/6J mice [15]. The results of the present study demonstrated that C3G induced similar hypoglycemic and hypolipidemic effects to SBp containing a comparable amount of C3G in HFHS diet-induced insulin-resistant mice. The findings of the present study are consistent with the previous studies on the effects of Saskatoon berry and C3G in glucose and lipid metabolism reported by our and other groups $[11,20,23]$. The findings suggest that $C 3 G$ potentially plays a critical role in the metabolic benefits of SBp in glucose and lipid metabolism.

A recent study demonstrated that $\mathrm{C} 3 \mathrm{G}$ administration normalized gut dysbiosis induced by a food contaminant, 3-chloro-1,2-propanediol, in rats, which was characterized by increased abundances of Lachnospiraceae and Actinobaceria phylum bacteria compared to 3-chloro-1,2-propanediol alone [25]. C3G is relatively stable in stomach and intestine [26], and it is converted to active metabolites, protocatechuic, vanillic and $p$-coumaric acids in gastrointestinal rats with the assistance from gut microbiota [27]. The present study demonstrated that C3G inhibited HFHS diet-induced gut dysbiosis in mice. Both C3G and SBp significantly increased the abundance of Bacteroidetes phylum bacteria and Muribaculaceae family bacteria. Muribaculaceae is a newly re-named family of bacteria under phylum Bacteroidetes, previously known as S24-7 or Homeothermaceae family. Over 600 species of Muribaculaceae family bacteria were inferred. Mouse was the most common host of Muribaculaceae, but it was also found in human, pig and non-human primates [28]. The functions of Muribaculaceae family bacteria have been mainly studied in mice to date. Muribaculaceae negatively influenced cellular processes involved in the development of chronic inflammatory bowel disease in mice [29]. An anti-diabetic agent, Acarbose, elongated lifespan in mice, which was associated with an increased abundance of Muribaculaceae and propionate, a short-chain fatty acid (SCFA), in feces [30]. Reduced levels of SCFA and relevant bacteria in the gut were associated with T2D patients and reversed by a high-fiber diet [31]. The findings of the present study suggest that Muribaculaceae family bacteria may be the targets of C3G and SBp in gut microbiota, which may contribute to the beneficial effects of C3G or SBp on metabolism and inflammation in mice. 
Although the impact of supplementation of C3G and SBp on the relative abundances of gut Bacteroidetes phylum bacteria and Muriculaceae family bacteria in HFHS diet-fed mice was comparable, the effects of $\mathrm{C} 3 \mathrm{G}$ on the compositions of 8 families of gut bacteria were significantly different from that associated with SBp (Figure 7A). For example, the relative abundance of Clostriaceae 1 was significantly increased in the C3G group compared to that in the HFHS group, but its relative abundance was not significantly different between the SBp and HFHS groups (Figure 8). The results suggest that both SBp and C3G supplementation upregulated the relative abundance of Muriculaceae family bacteria, a type of gut bacteria negatively correlated with diabetes and inflammation, in mice gut, but their effects on the regulation of other family bacteria were not consistent, which indicates that the difference in other family bacteria may result from other components in SBp.

Functional prediction analysis helps to predict the potential of changes in gut microbiome based on metabolic pathways. Previous studies demonstrated that a high-fat diet reduced lipid metabolism, including short-chain fatty acids and the substrates for hepatic gluconeogenesis [32]. A recent study found that strawberry supplementation induced marked changes in functional potentials of microbial composition in $\mathrm{db} / \mathrm{db}$ mice [33]. The present study has demonstrated that the HFHS diet upregulated the genes of gut microbes with predicted functions related to inflammation and downregulated microbial genes related to lipids, carbohydrates, amino acid, cofactors and vitamins. SBp or C3G supplementation to the HFHS diet tends to normalize the impact of the HFHS diet on metabolism and inflammation in gut microbiota in mice. We noticed that the HFHS diet increased the abundance of bacterial genes related to transcription but decreased the genes of bacteria contributing to protein translation in mice, which possibly results from the inhibitory effect of the HFHS diet on the translation of genes related to the metabolism, such as enzymes and cofactors. SBp and C3G supplementation neutralized the contradictory effects of the HFHS diet on microbial genes related to transcription and translation in mice (Figure 8). The outcome of the functional prediction supports the metabolic and inflammatory changes induced by the HFHS diet and the resilient capacity of SBp or C3G in metabolism in HFHS diet-fed mice in the present study.

\section{Conclusions}

The present study demonstrated that C3G supplementation resulted in similar beneficial effects compared to SBp containing an equal amount of C3G on HFHS diet-induced disorders in metabolism, inflammation and gut microbiota in mice. The results of functional predication analysis indicated that $C 3 G$ reversed the changes in microbe genes related to metabolism and inflammation induced by the HFHS diet in mice. The findings suggest that C3G and SBp are potential prebiotics, which may mitigate Western diet-induced disorders in metabolism, inflammation and gut dysbiosis. The safety and proper dosage of $\mathrm{C} 3 \mathrm{G}$ in humans remain to be determined. Saskatoon berry is a recognized fruit and can be used as a functional fruit or food supplement. The results of the present study may help to design clinical studies to investigate the efficacy of C3G and functional foods containing high amounts of C3G, such as Saskatoon berry, for the prevention and management of T2D in humans.

Declarations: Ethics approval and consent: The animal experimental protocols have been approved by the Animal Protocol and Ethics Committee in the University of Manitoba (AC-11328, approved on 27 September 2017). R.Z. and F.H., a graduate student, received required training and his involvement in the animal experiments has been approved. Consent for human data is not applicable to the present study.

Author's Information: F.H. is a graduate student in the Department of Food and Human Nutritional Sciences in the University of Manitoba, supervised by G.X.S.

Author Contributions: F.H. contributed to bioinformatics analysis of microbiome data, manuscript preparation and sample collection from animals; R.Z. contributed to animal experiments and biochemical analyses; M.X. provided important reagents and contributed to experiment design; G.X.S. contributed to project design, supervision and manuscript writing. All authors have read and agreed to the published version of the manuscript.

Funding: The experiments were financially supported by an operating grant from Diabetes Canada to G.X.S. (OG-3-15-4889-GS). 
Acknowledgments: The authors are grateful to the University of Manitoba for partial support of the stipend of F.H. through Graduates Enhancement of Tri-Council Stipends.

Conflicts of Interest: The authors declare that they0 have no competing interests.

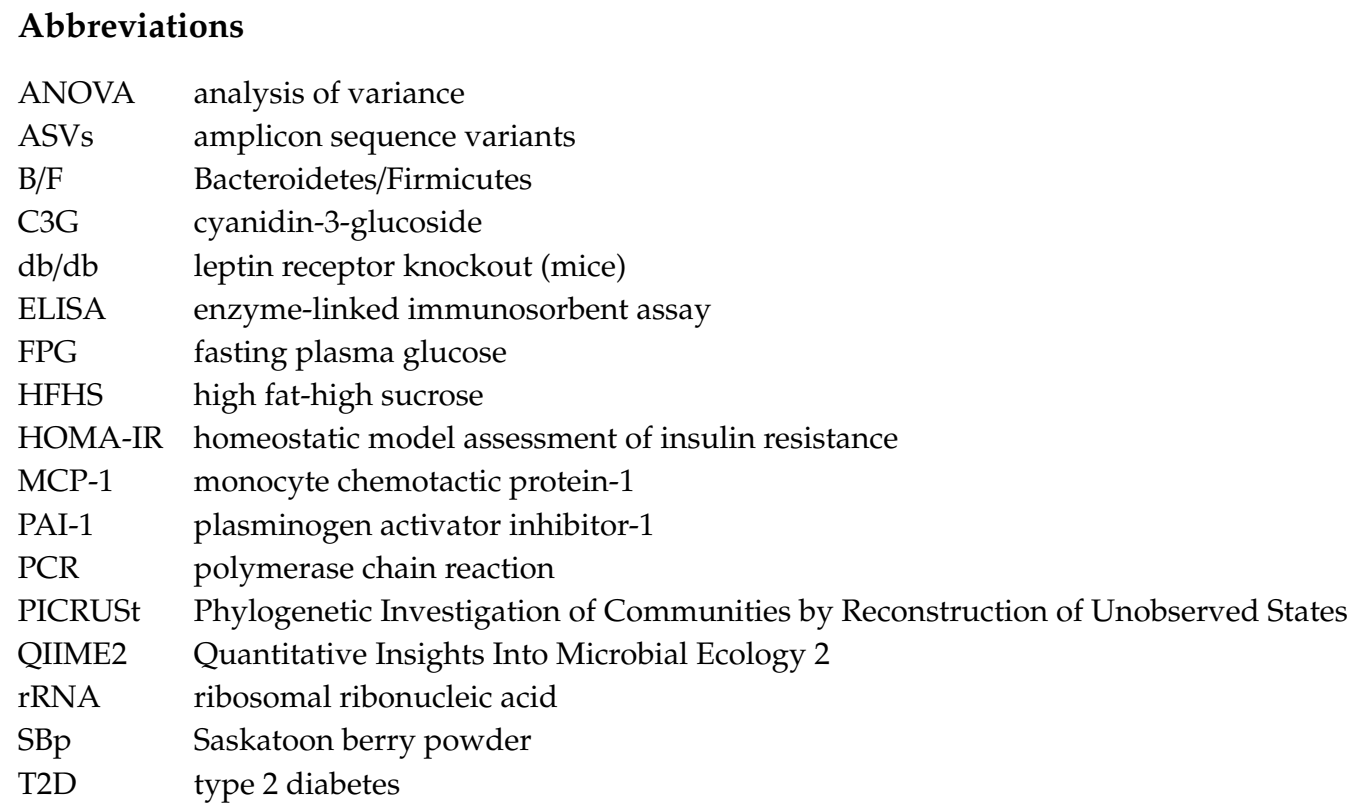

\section{References}

1. Henning, R.J. Type-2 diabetes mellitus and cardiovascular disease. Future Cardiol. 2018, 14, 491-509. [CrossRef] [PubMed]

2. Skyler, J.S.; Bakris, G.L.; Bonifacio, E.; Darsow, T.; Eckel, R.H.; Groop, L.; Groop, P.-H.; Handelsman, Y.; Insel, R.A.; Mathieu, C. Differentiation of diabetes by pathophysiology, natural history, and prognosis. Diabetes 2017, 66, 241-255. [CrossRef] [PubMed]

3. Bradley, B.H.R. Dietary fat and risk for type 2 diabetes: A review of recent research. Cur. Nutr. Rep. 2018, 7, 214-226. [CrossRef] [PubMed]

4. Kopp, W. How western diet and lifestyle drive the pandemic of obesity and civilization diseases. Diab. Met. Syn. Obes. Targets Ther. 2019, 12, 2221. [CrossRef] [PubMed]

5. Sonnenburg, J.L.; Bäckhed, F. Diet-microbiota interactions as moderators of human metabolism. Nature 2016, 535, 56-64. [CrossRef] [PubMed]

6. Wen, L.; Duffy, A. Factors influencing the gut microbiota, inflammation, and type 2 diabetes. J. Nutr. 2017, 147, 1468S-1475S. [CrossRef]

7. Mazza, G. Compositional and functional properties of saskatoon berry and blueberry. Int. J. Fruit. Sci. 2005, 5, 101-120. [CrossRef]

8. Mazza, G. Anthocyanins and Other Phenolic Compounds of Saskatoon Berries Amelanchier alnifolia Nutt. J. Food Sci. 1986, 51, 1260-1264. [CrossRef]

9. Gould, K.; Smreciu, A.; Wood, S. Amelanchier Alnifolia: Saskatoon, Service Berry, June Berry, Shadbush, Shadblow, Indian Pear, May Cherry. Available online: https://era.library.ualberta.ca/items/d3680caa-0ad343cc-abe4-cf398296ae9b (accessed on 13 August 2020).

10. Hosseinian, F.S.; Beta, T. Saskatoon and wild blueberries have higher anthocyanin contents than other Manitoba berries. J. Agric. Food Chem. 2007, 55, 10832-10838. [CrossRef]

11. Zhao, R.; Le, K.; Li, W.; Ren, S.; Moghadasian, M.H.; Beta, T.; Shen, G.X. Effects of Saskatoon berry powder on monocyte adhesion to vascular wall of leptin receptor-deficient diabetic mice. J. Nutr. Biochem. 2014, 25, 851-857. [CrossRef]

12. De Souza, D.R.; Willems, J.L.; Low, N.H. Phenolic composition and antioxidant activities of saskatoon berry fruit and pomace. Food Chem. 2019, 290, 168-177. [CrossRef] [PubMed] 
13. Lachowicz, S.; Oszmiański, J.; Seliga, Ł.; Pluta, S. Phytochemical composition and antioxidant capacity of seven saskatoon berry (Amelanchier alnifolia Nutt.) Genotypes Grown in Poland. Molecules 2017, $22,853$. [CrossRef] [PubMed]

14. Zhao, R.; Xie, X.; Le, K.; Li, W.; Moghadasian, M.H.; Beta, T.; Shen, G.X. Endoplasmic reticulum stress in diabetic mouse or glycated LDL-treated endothelial cells: Protective effect of Saskatoon berry powder and cyanidin glycans. J. Nutr. Biochem. 2015, 26, 1248-1253. [CrossRef] [PubMed]

15. Zhao, R.; Khafipour, E.; Sepehri, S.; Huang, F.; Beta, T.; Shen, G.X. Impact of Saskatoon berry powder on insulin resistance and relationship with intestinal microbiota in high fat-high sucrose diet-induced obese mice. J. Nutr. Biochem. 2019, 69, 130-138. [CrossRef]

16. Miranda, C.L.; Johnson, L.A.; De Montgolfier, O.; Elias, V.D.; Ullrich, L.S.; Hay, J.J.; Paraiso, I.L.; Choi, J.; Reed, R.L.; Revel, J.S. Non-estrogenic xanthohumol derivatives mitigate insulin resistance and cognitive impairment in high-fat diet-induced obese mice. Sci. Rep. 2018, 8, 1-17. [CrossRef]

17. Walters, W.; Hyde, E.R.; Berg-Lyons, D.; Ackermann, G.; Humphrey, G.; Parada, A.; Gilbert, J.A.; Jansson, J.K.; Caporaso, J.G.; Fuhrman, J.A. Improved bacterial 16S rRNA gene (V4 and V4-5) and fungal internal transcribed spacer marker gene primers for microbial community surveys. Msystems 2016, 1, e00009-e00015. [CrossRef]

18. Bolyen, E.; Rideout, J.R.; Dillon, M.R.; Bokulich, N.A.; Abnet, C.C.; Al-Ghalith, G.A.; Alexander, H.; Alm, E.J.; Arumugam, M.; Asnicar, F. Reproducible, interactive, scalable and extensible microbiome data science using QIIME 2. Nat. Biotech. 2019, 37, 852-857. [CrossRef]

19. Langille, M.G.; Zaneveld, J.; Caporaso, J.G.; McDonald, D.; Knights, D.; Reyes, J.A.; Clemente, J.C.; Burkepile, D.E.; Thurber, R.L.V.; Knight, R. Predictive functional profiling of microbial communities using 16S rRNA marker gene sequences. Nat. Biotech. 2013, 31, 814. [CrossRef]

20. Yamashita, Y.; Wang, L.; Nanba, F.; Ito, C.; Toda, T.; Ashida, H. Procyanidin promotes translocation of glucose transporter 4 in muscle of mice through activation of insulin and AMPK signaling pathways. PLoS ONE 2016, 11, e0161704. [CrossRef]

21. Luna-Vital, D.A.; de Mejia, E.G. Anthocyanins from purple corn activate free fatty acid-receptor 1 and glucokinase enhancing in vitro insulin secretion and hepatic glucose uptake. PLoS ONE 2018, 13, e0200449. [CrossRef]

22. Du, C.; Shi, Y.; Ren, Y.; Wu, H.; Yao, F.; Wei, J.; Wu, M.; Hou, Y.; Duan, H. Anthocyanins inhibit high-glucose-induced cholesterol accumulation and inflammation by activating LXR $\alpha$ pathway in HK-2 cells. Drug Des. Dev. Ther. 2015, 9, 5099.

23. Sun, C.-D.; Zhang, B.; Zhang, J.-K.; Xu, C.-J.; Wu, Y.-L.; Li, X.; Chen, K.-S. Cyanidin-3-glucoside-rich extract from Chinese bayberry fruit protects pancreatic $\beta$ cells and ameliorates hyperglycemia in streptozotocin-induced diabetic mice. J. Med. Food 2012, 15, 288-298. [CrossRef] [PubMed]

24. Xie, X.; Zhao, R.; Shen, G.X. Influence of delphinidin-3-glucoside on oxidized low-density lipoprotein-induced oxidative stress and apoptosis in cultured endothelial cells. J. Agric. Food Chem. 2012, 60, 1850-1856. [CrossRef] [PubMed]

25. Chen, G.; Wang, G.; Zhu, C.; Jiang, X.; Sun, J.; Tian, L.; Bai, W. Effects of cyanidin-3-O-glucoside on 3-chloro-1, 2-propanediol induced intestinal microbiota dysbiosis in rats. Food Chem. Toxicol. 2019, 133, 110767. [CrossRef]

26. Pradipta, I.; Kong, D.; Tan, J.B.L. Natural organic antioxidants from green tea form a protective layer to inhibit corrosion of steel reinforcing bars embedded in mortar. Constr. Build. Mater. 2019, 221, 351-362. [CrossRef]

27. Chen, Y.; Li, Q.; Zhao, T.; Zhang, Z.; Mao, G.; Feng, W.; Wu, X.; Yang, L. Biotransformation and metabolism of three mulberry anthocyanin monomers by rat gut microflora. Food Chem. 2017, 237, 887-894. [CrossRef]

28. Lagkouvardos, I.; Lesker, T.R.; Hitch, T.C.A.; Gálvez, E.J.C.; Smit, N.; Neuhaus, K.; Wang, J.; Baines, J.F.; Abt, B.; Stecher, B.; et al. Sequence and cultivation study of Muribaculaceae reveals novel species, host preference, and functional potential of this yet undescribed family. Microbiome 2019, 7, 28. [CrossRef]

29. Volk, J.K.; Nyström, E.E.; van der Post, S.; Abad, B.M.; Schroeder, B.O.; Johansson, Å.; Svensson, F.; Jäverfelt, S.; Johansson, M.E.; Hansson, G.C. The Nlrp6 inflammasome is not required for baseline colonic inner mucus layer formation or function. J. Exp. Med. 2019, 216, 2602-2618. [CrossRef] 
30. Smith, B.J.; Miller, R.A.; Ericsson, A.C.; Harrison, D.C.; Strong, R.; Schmidt, T.M. Changes in the gut microbiome and fermentation products concurrent with enhanced longevity in acarbose-treated mice. BMC Microbiol. 2019, 19, 130. [CrossRef]

31. Zhao, L.; Zhang, F.; Ding, X.; Wu, G.; Lam, Y.Y.; Wang, X.; Fu, H.; Xue, X.; Lu, C.; Ma, J.; et al. Gut bacteria selectively promoted by dietary fibers alleviate type 2 diabetes. Science 2018, 359, 1151-1156. [CrossRef]

32. Zhang, L.; Xie, C.; Nichols, R.G.; Chan, S.H.; Jiang, C.; Hao, R.; Smith, P.B.; Cai, J.; Simons, M.N.; Hatzakis, E. Farnesoid $\mathrm{X}$ receptor signaling shapes the gut microbiota and controls hepatic lipid metabolism. MSystems 2016, 1, e00016-e00070. [CrossRef] [PubMed]

33. Petersen, C.; Wankhade, U.D.; Bharat, D.; Wong, K.; Mueller, J.E.; Chintapalli, S.V.; Piccolo, B.D.; Jalili, T.; Jia, Z.; Symons, J.D. Dietary supplementation with strawberry induces marked changes in the composition and functional potential of the gut microbiome in diabetic mice. J. Nutr. Biochem. 2019, 66, 63-69. [CrossRef] [PubMed]

(C) 2020 by the authors. Licensee MDPI, Basel, Switzerland. This article is an open access article distributed under the terms and conditions of the Creative Commons Attribution (CC BY) license (http://creativecommons.org/licenses/by/4.0/). 